CUADERNOS DE ESTUDIOS GALLEGOS, LXI Núm. 127 (enero-diciembre 2014), págs. 251-278

ISSN: $0210-847 \mathrm{X}$

DOI: $10.3989 /$ ceg.2014.127.08

\title{
LA ESCUELA DE CIEGOS DEL CAMPO DE LA LEÑA, A CORUÑA: LOS INICIOS DE LA ENSEÑANZA ESPECIAL EN GALICIA
}

ANA RoDRÍGuez DÍAZ

Universidad Nacional de Educación a Distancia (UNED) 


\title{
LA ESCUELA DE CIEGOS DEL CAMPO DE LA LEÑA, A CORUÑA: LOS INICIOS DE LA ENSEÑANZA ESPECIAL EN GALICIA
}

\section{RESUMEN}

Este artículo pretende mostrar la relevancia de la escuela de ciegos instaurada en la ciudad de A Coruña en el año 1895, por parte del presbítero José María Salgado. Esta escuela representa uno de los escasos establecimientos que, a finales del siglo XIX y principios del XX, se dedican en España a la educación de estos alumnos. Igualmente, constituye una contribución significativa en la historia de la enseñanza especial gallega. La escuela de Salgado figura como la segunda institución destinada a la instrucción de ciegos que se establece en Galicia y la primera que se establece en la ciudad de A Coruña. El presente estudio, abordado desde una perspectiva histórica, descriptiva y comparada, se ha fundamentado en fuentes archivísticas concretas, así como en la prensa histórica gallega y otras publicaciones históricas y actuales relativas a la educación especial. La indagación bibliográfica efectuada para el desarrollo del trabajo constata la escasez de estudios monográficos existentes sobre estas escuelas inauguradas a finales del siglo XIX. Las lagunas de información son grandes, lo mismo que el olvido al que han quedado relegados algunos de estos establecimientos, que han representado un hito en los inicios de la institucionalización de la enseñanza especial.

Palabras Clave: Escuela de ciegos, enseñanza especial, José María Salgado, A Coruña.

\section{A ESCOLA DE CEGOS DO CAMPO DA LEÑA: OS INICIOS DO ENSINO ESPECIAL EN GALICIA}

\section{Resumo}

Este artigo pretende mostrar a relevancia da escola de cegos instaurada na cidade da Coruña no ano 1895 polo presbítero José María Salgado. Esta escola representa un dos poucos establecementos que, a finais do século XIX e principios do XX, se dedican en España á educación destes alumnos. Igualmente, constitúe unha contribución significativa na historia do ensino especial galego. A Escola de Salgado figura como a segunda institución destinada á instrución de cegos, que se establece en Galicia, e a primeira que se establece na cidade da Coruña. O presente estudo abordado dende unha perspectiva histórica, descritiva e comparada, fundamentouse en fontes arquivísticas concretas, así como na prensa histórica galega e outras publicacións históricas e actuáis relativas á educación especial. A indagación bibliográfica efectuada para o desenvolvemento do traballo constata a escaseza de estudos monográficos existentes sobre os Colexios e Escolas inaugurados a finais do século XIX.

As lagoas de información son grandes, o mesmo que o esquecemento a que quedaron relegados algúns destes establecementos, que representaron un fito nos inicios institucionalización do ensino especial. Palabras Clave: Escola de cegos, ensino especial, José María Salgado, A Coruña.

\section{SCHOOL FOR THE BLIND, CAMPO DE LA LEÑA, A CORUÑA: THE BEGINNINGS OF SPECIALIST EDUCATION IN GALICIA}

\begin{abstract}
The aim of this article is to show the relevance of the school for the blind opened in A Coruña by the Presbyterian José María Salgado, in 1895. This school was one of the few establishments in Spain that was dedicated, to the education these students, in the late $19^{\text {th }}$ and early $20^{\text {th }}$ century. It also constitutes a significant contribution to the history of specialist education in Galicia. The Salgado School was the second institution for the blind of Galicia and the second in the city of A Coruña. This study approached Specialist Education from an historic, descriptive and comparative perspective, is based on concrete archived sources, along with the Galician press and other relevant current and historic publications. The literature review carried out to develop this work found very few monographic studies on these schools inaugurated at the end of the $19^{\text {th }}$ century. There are large gaps in the information. Although they are often overlooked, some of these establishments represented a milestone in the beginnings of the institutionalisation of specialist education.
\end{abstract}

KEY wORDS: School for the blind, specialist education, José María Salgado, A Coruña. 
Recibido/Received: 21/06/2014

Aceptado/Accepted: 11/08/2014

LAS INSTITUCiOnES DE ENSEÑANZA ESPECIAL EN ESPAÑA A FINALES DEL SIGLO XIX

finales del siglo XIX, tanto en España como en Europa, se establecie-
ron instituciones específicas para el tratamiento y educación de aquellos
alumnos que por sus necesidades especiales no podían beneficiarse de la enseñanza ordinaria. Las escuelas y colegios dedicados a la enseñanza de los ciegos, al igual que la de sordomudos, comenzaron su funcionamiento con anterioridad a los que acogen a los menores con otro tipo de deficiencias.

El primer proyecto de crear una escuela para ciegos lo promovió la Sociedad Económica Matritense en el año 1835. El proyecto resultó aprobado, pero no obtuvo subvención. "No obstante se autoriza la enseñanza de ciegos en una sección del Colegio Nacional de Sordomudos a la sazón Colegio de Sordomudos". En el año 1841, la enseñanza de los ciegos dispondrá de subvención pública.

En cualquier caso, la justa petición de que todos los alumnos recibiesen la enseñanza primaria, independiente de su condición, comenzó a adquirir visos de realidad en España a raíz de la Ley de Instrucción Pública de 1857, que estipulaba la creación de un establecimiento dedicado a la enseñanza especial en cada uno de los distritos universitarios. El artículo 108 de la ley determinaba el deber del Gobierno de promover las enseñanzas para los sordomudos y ciegos, procurando que hubiera por lo menos una escuela de esta clase en cada distrito universitario ${ }^{2}$.

El contexto histórico-social en que nacieron estas instituciones está marcado por una mentalidad general de ignorancia, pesimismo, repulsión y rechazo hacia lo que se considera "anormal". La mayoría de las publicaciones, discursos o conferencias sobre los que padecían algún tipo de deficiencia están salpicados de términos y connotaciones de menosprecio. Es común que se les mencione como

\footnotetext{
1 Antonio Aguado Díaz, Historia de las deficiencias, Madrid, Once, 1995, págs. 102-103.

2 Francisco Fernández Villabrille, Estado actual y organización de la enseñanza de Sordomudos y ciegos. Memoria dirigida al Ministro de Fomento, Madrid, Imprenta del Colegio de Sordomudos, 1862.
} 
infelices, miserables, privados de la luz del sentir, del conocimiento, o como seres embrutecidos. La ley Moyano se refería a ellos como "pobres desgraciados"3. De acuerdo con esta mentalidad de exclusión, podría considerarse a los colegios y escuelas que se iban inaugurando a finales del siglo XIX como centros benéficos, movidos por la caridad y compasión, con una misión redentora más que instructiva. Sin embargo, de "puertas adentro", la intención educativa estaba perfectamente explícita en reglamentos y objetivos.

La ley no llegó a hacerse efectiva en la práctica. En el año 1900 solo las ciudades de Madrid, Barcelona, Santiago de Compostela, Burgos, Zaragoza, Sevilla, Valencia, Deusto, Oviedo y Córdoba contaban con un colegio de enseñanza especial que atendía conjuntamente a ciegos y sordomudos. En cuanto a las escuelas, se encontraban la de Carabanchel, Alicante, Salamanca, Tarragona, Badajoz y Madrid ${ }^{4}$. A todo esto habría que añadir que la escuela de ciegos de A Coruña se encontraba funcionando desde hacía cinco años.

En el transcurso de las tres primeras décadas del siglo XX, el panorama institucional de centros de sordomudos y ciegos seguía siendo desolador: las escuelas y colegios existentes continuaban siendo escasos, y todavía había muchas ciudades españolas donde no existía ningún centro (como en las ciudades de Álava, Albacete, Almería, Ávila, Baleares, Burgos, Cáceres, Canarias, Ciudad Real, Córdoba, Cuenca, Gerona, Guadalajara, Huelva, Huesca, Jaén, León, Logroño, Lugo, Murcia, Navarra, Orense, Palencia, Pontevedra, Segovia, Soria, Tarragona, Teruel, Toledo o Zamora ${ }^{5}$ ).

Como bien dice el historiador Gascón Ricao ${ }^{6}$, no existe ningún estudio monográfico (o si existen son escasísimos) sobre las escuelas que se fueron inaugurando en las distintas capitales españolas durante la segunda mitad del siglo XIX, ni estudios con referencia a los maestros y métodos. Aún así, abundan las memorias, conferencias y discursos publicados por los maestros de ciegos más

\footnotetext{
3 Ángel Serafín Porto Ucha, "La educación especial en la formación de maestros y maestras en el primer tercio del siglo XX. Algunos datos referentes a Galicia", El largo camino hacia una educación inclusiva. La educación especial y social del siglo XIX a nuestros días, XV Coloquio de Historia de la Educación, Pamplona-Iruñea, Universidad de Navarra, 2009, pág. 337.

4 Pedro Molina Martín, "Los Institutos de sordomudos y ciegos", La Escuela Moderna: revista pedagógica hispano-americana, 111 (junio de 1900), pág. 455.

5 Datos de la estadística publicada por el Patronato Nacional de Sordomudos y Ciegos sobre las escuelas y colegios dedicadas a la enseñanza de los mismos, recogida de El noticiero gallego: semanario destinado a fomentar los intereses morales y materiales del Magisterio de primera enseñanza, 1542 (11 de junio de 1925), pág. 1.

6 Antonio Gascón RicaO, "Crónica simple sobre diez años de investigación sobre las personas sordas", en Personas sordas y Universidad: el valor de la docencia y de la investigación universitarias. Conferencia presentada en las II Jornadas Universitarias organizadas por la Universidad de Sevilla, 15 de abril de 2009, Sevilla, Universidad de Sevilla, 2009, págs. 254-261.
} 
relevantes de la época, entre otros, Ballesteros, López Navalón y Nebreda. La información existente apunta a que los escasos centros establecidos de acuerdo a las disposiciones legislativas fueron la mayoría muy pobres en el sentido educativo. Si bien es cierto que unos contaban con mejores recursos que otros, solo el Colegio de sordomudos y ciegos de Madrid disfrutaba de instalaciones, profesorado y material adecuado, además de capacidad para un número de alumnos idóneo, tal como dictaban las nuevas corrientes europeas ${ }^{7}$.

El atraso que existía en España en materia de educación especial era muy grande si se compara con otras ciudades europeas. Pero este hecho no es de extrañar, siendo España un país pobre, de escasa industrialización. Políticamente, no existía solidez, y era notorio el desinterés por la cultura, lo que derivaba en una escasa instrucción pública y un alto índice de analfabetismo ${ }^{8}$.

La situación escolar que presentaba Galicia en las últimas décadas del siglo XIX era más deficitaria que la que sufren el resto de las provincias españolas. En general, todos los recursos educativos materiales y humanos eran menores en Galicia: menos maestros, menor número de escuelas, peor calidad de estas y baja asistencia escolar'. Entre las causas que tratan de explicar esta diferencia se encuentran una población dispersa, diseminada, que se desatiende de todo lo relativo a la instrucción, y un Estado centralizado que se despreocupa de la periferia ${ }^{10}$. Sin embargo - y pese a ello- Galicia se sentirá avivada por la misma inquietud pedagógica que el resto de España y, junto con la aparición de un incipiente provincialismo que luchaba por proteger su identidad, no faltaron los discursos filantrópicos, lo mismo que la acción social en favor a la asistencia y enseñanza de los más desfavorecidos. Tanto es así que, tras un repaso histórico de la evolución en la educación, el nombre de Galicia parece haber quedado estrechamente vinculado a hechos, instituciones y figuras de enorme transcen-

\footnotetext{
7 Mariano Herraiz Gascuella, Aproximación a la educación especial española del primer tercio del siglo XX, Murcia, Compobell, 1995, pág. 171.

8 Manuel Puelles Benítez, "Los valores en las grandes leyes de educación", en Mercedes Muñoz Repiso, Javier M. Valle López, José Luis Villalaín (eds.), Educación y valores en España, Madrid, Ministerio de Educación y Ciencia, CIDE, 1992, págs. 63-83. En el mismo sentido: Olegario Negrín FaJARdo, Historia de la educación española, Madrid, Universidad Nacional de Educación a Distancia, 2011; José L. Comellas García, Historia España Contemporánea, Madrid, RIALP, 2002.

9 Narciso de Gabriel Fernández, "Financiación de la escuela pública en la España del siglo XIX. El caso Gallego", Historia de la educación, revista interuniversitaria, 7 (1988), págs. 163 178.

${ }^{10}$ Mercedes SuÁrez Pazos, "El campesinado gallego y su rechazo a la escuela primaria (18681874)", Historia de la educación. Revista interuniversitaria, 2 (1983) págs. 317-324. También es interesante consultar: Antón Costa Rico, Historia da educación e da cultura en Galicia, (Séculos IV-XX), Vigo, Xerais, 2004; Manuel VeIga, O pacto galego na construcción de España, Vigo, A nosa terra, 2003.
} 
dencia para el futuro de la historia de la educación especial española, e incluso del extranjero.

Cabe resaltar que a Galicia le corresponde el privilegio de haber sido la primera comunidad de España en tener un colegio para sordomudos y ciegos de acuerdo a los mandatos de la Ley de Instrucción Pública. Así lo declaraba Manuel López Návalón, su primer director, en una de las memorias que redacta sobre el Colegio:

Galicia, cuya estadística de sordomudos y de ciegos es muy numerosa debida en parte a la montuosidad de su país y a otras muchas causas locales, ha sido la primera que ha llevado al terreno práctico la realización de tan filantrópico pensamiento ${ }^{11}$.

Además, es importante señalar que la organización del Colegio regional gallego era digna de destacar, si se compara con los establecimientos contemporáneos ${ }^{12}$. Sin embargo, como se desprende de lo debatido en el Congreso Pedagógico celebrado en Pontevedra en el año 1887, este colegio no era suficiente para atender a la numerosa población de sordomudos y ciegos contabilizada en Galicia. Victoriano Encinas Reyes, que entonces era segundo maestro interino en la Escuela Normal de Pontevedra, se lamentaba del escaso número de colegios y escuelas, manifestando que llevar a la práctica la enseñanza de los alumnos ciegos y sordomudos era un principio humanitario ineludible y una obligación del Estado:

Es, pues, una de las principales obligaciones que el estado tiene, la de atender a la educación e instrucción de estos hermanos nuestros, tanto más, cuanto que su desgracia es inmensa y su situación social tristísima cual ninguna. Podríais objetarme que hay Escuelas especiales para educar e instruir a estos desgraciados, y seguramente cuando tal objeción me hicierais, olvidabais el corto número que de ellas existe o no teníais en consideración el crecidísimo que hay de sordo-mudos y ciegos. Galicia nos ofrece un ejemplo, en este asunto, que evita todo género de razonamientos. Para sus cuatro provincias, que yo sepa, no tiene más que un

\footnotetext{
${ }^{11}$ Manuel López Navalón, Memoria sobre el estado actual del Colegio de Sordo-Mudos y de Ciegos de Santiago. Mejoras de que es susceptible para su futuro progreso y prosperidad. Memoria, Santiago de Compostela, Imprenta Manuel Miras, 1867, pág. 19.

${ }^{12}$ José A. Díaz Huertas, Miguel A. Ruiz Díaz y Víctor Ruiz Díaz, Infancia y discapacidad, Madrid, Sociedad de Pediatría Social, 2007, pág. 11.
} 
Colegio establecido en Santiago. Cada una de estas provincias si mal no recuerdo, tiene derecho a diez plazas anuales, en el citado Colegio - cinco de ciegos y cinco de sordo-mudos- total 40 entre unos y otros $^{13}$.

El Colegio de Santiago de Compostela se inauguró el 1 de junio de 1864. Según se recogía en su Reglamento ${ }^{14}$, mantenía a 40 alumnos internos, con fondos de la escuela, y había 20 plazas más que eran financiadas por las corporaciones o particulares. Sin embargo, las plazas disponibles no eran suficientes. En el año 1985, el presbítero José María Salgado instauró en A Coruña la primera escuela de ciegos:

Es de sentir que institución tan benemérita, segunda en Galicia, pues solo funciona la escuela de sordomudos y ciegos de Santiago, no obtenga mayor protección del pueblo y de las autoridades ${ }^{15}$.

En años anteriores había habido algún que otro intento, por parte del Ayuntamiento, de establecer una escuela normal para ciegos, pero parece ser que la propuesta no llegó a prosperar. Esto es lo que consta en las actas de la ciudad, del 28 de abril del año 1835:

En este Ayuntamiento, con presencia de la Real orden de 2 del corriente que el Señor Gobernador Civil comunica en el 22, por medio del Boletín Oficial $n .^{\circ} 188$ relativo al establecimiento de la Escuela Normal de ciegos en que se propone enseñarles a leer, escribir y contar, previniendo que para que esta medida sea extensiva a los habitantes de esta Provincia se le dé una relación de los ciegos que haya en este Pueblo, expresiva de la Calidad, sexo de cada uno y de los que tengan por si recursos para atender su subsistencia, acordó la ciudad se encargue esta relación a los Alcaldes de Barrio, para que tomando indicación de los celadores de

\footnotetext{
${ }^{13}$ Eugenio Manuel Otero Urtaza, "O discurso de Victoriano Encinas no Congreso Pedagóxico de Pontevedra (1887)", Sarmiento. Anuario Galego de Historia da Educación, 1 (1997), págs. 119214.

${ }^{14}$ Colegio de Sordomudos y Ciegos de Santiago de Compostela, Bases y Reglamento para el establecimiento y régimen del colegio de Sordomudos y de ciegos del distrito universitario de Santiago, Imprenta Manuel Mirás, 1863, pág. 10.

15 "Escuela de ciegos de la Coruña", Revista gallega: semanario de literatura e intereses regionales, 120 (27 de junio de 1987), pág. 4.
} 
los mismos dentro de seis días, presenten al ayuntamiento dichas relaciones con las circunstancias determinadas ${ }^{16}$.

En cualquier caso, en las primeras décadas del XX — según los datos que figuran en la estadística de colegios y escuelas públicas y privadas encargadas de la educación de ciegos y sordomudos - el panorama no había cambiado. En Galicia, en el año 1925, había solamente dos instituciones que hacían frente a la enseñanza de estos alumnos, las mismas que las inauguradas en el siglo anterior: el Colegio regional de sordomudos y ciegos de Santiago de Compostela y la Escuela de ciegos y niños pobres del Campo de la leña, que, por entonces, contaba con 12 alumnos externos y dependía del Patronato de las Escuelas Populares Gratuitas ${ }^{17}$.

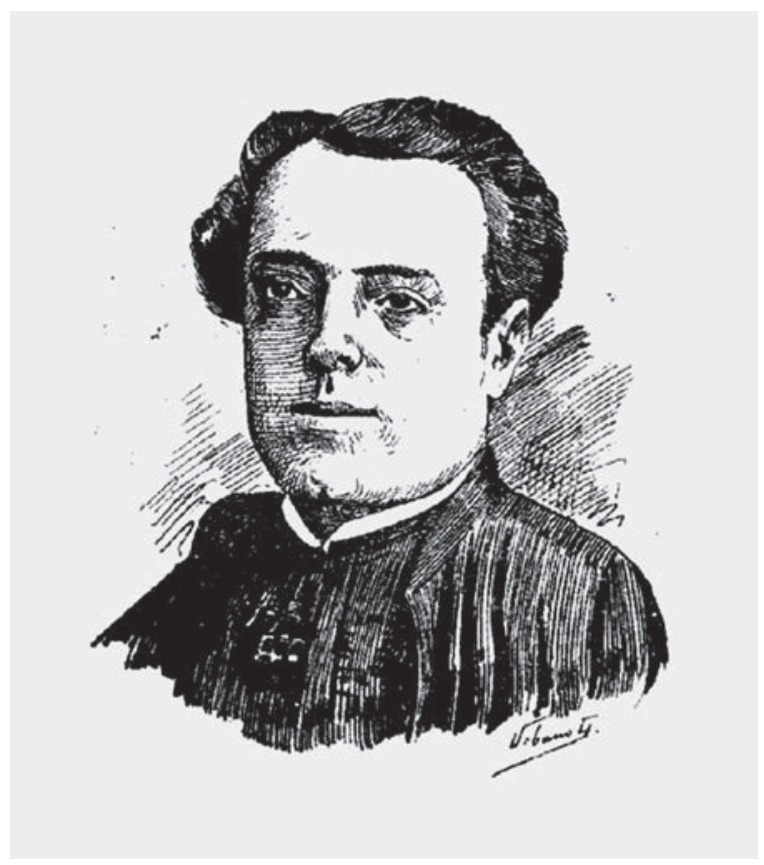

Fig. $1^{18}$

\footnotetext{
${ }^{16}$ José Manuel Fernández CaAmaño, La Coruña Vista Desde sus Libros de Actas II, Tomo II, Madrid, Editorial Visión Net, 2006, pág. 111.

17 "Patronato nacional de sordomudos y ciegos", El Noticiero gallego: semanario destinado a fomentar los intereses morales y materiales del Magisterio de primera enseñanza, 1542 (11 de junio de 1925), pág. 1.

${ }^{18}$ Uno de los pocos retratos que aparecen de Salgado, se encuentra en la portada de Don Pepito: Semanario satírico y político, 89 (23 de noviembre de 1890).
} 


\title{
El presbítero José María Salgado, Fundador de la Escuela de CIEGOS DE A CORUÑA
}

El nombre de José María Salgado se cita, junto con el nombre de otros ilustres gallegos bienhechores de la patria, en un artículo publicado por la revista madrileña La Escuela Moderna. En dicho escrito se reconoce a Salgado como el piadoso erector de la Escuela de ciegos de La Coruña ${ }^{19}$. Pese a ese reconocimiento, es una personalidad sobre la que apenas se ha escrito nada. Sin embargo, tal como testimonia uno de los escasos artículos que aparecen sobre su persona, es mucho lo que habría que decir acerca de este ilustre sacerdote:

\begin{abstract}
Mucho podría decirse del Sr. Salgado como hombre de ciencia, sacerdote caritativo, e incansable defensor de nuestra querida España. Tengo a la vista infinidad de periódicos argentinos, que comprueban lo que ha el Sr. Salgado ha hecho en esos países por la ciencia, la religión y la prosperidad de nuestra nación ${ }^{20}$.
\end{abstract}

Según testimonia su certificado de bautismo, José María Salgado Ferreira fue bautizado en la ciudad de A Coruña, en la parroquia de San Nicolás, el día 17 de julio de 1840. Asimismo, en dicho documento figura el nombre de sus padres: Diego Salgado, natural de Santa María de Rasela, obispado de Orense, y Francisca Ferreiro, de Santa María de Oris, obispado de Lugo ${ }^{21}$.

En algún que otro artículo de publicaciones de finales del siglo XIX y principios del XX se encuentran detalles reveladores de la vida y obra de este filántropo $^{22}$. Salgado se vio obligado a alejarse del país, amenazado por los desórdenes políticos de la época, al igual que muchos otros gallegos que ocupaban destacados puestos de jefes y oficiales. Abandonó su puesto como capellán de artillería de A Coruña a raíz de la revolución del 68, dirigiéndose a América del Sur (concretamente, a Argentina). Poco después de su llegada al Nuevo Continente, fue

\footnotetext{
19 "Concepción Arenal en el aspecto pedagógico" en La Escuela Moderna: revista pedagógica y administrativa de primera de enseñanza, 414 (marzo de 1926), pág. 239.

20 "Nuestro grabado", Don Pepito: semanario satírico y político, 89 (23 de noviembre de 1890), pág. 2.

${ }^{21}$ Archivo Histórico Universitario de Santiago de Compostela (en adelante sólo AHUS), Fondo Universitario: colexios privados, Caixa, SH 228, Expediente 19, Escuela gratuita de ciegos y niños pobres, 1904.

${ }^{22}$ Entre ellos, el artículo citado con anterioridad, "Nuestro grabado", Don Pepito: semanario satírico y político, 89 (23 de noviembre de 1890), pág. 2, o "Don José María Salgado", firmado por JeCOUfer, en Aires da miña terra, 27 (8 de noviembre de 1908). No obstante, los detalles biográficos se repiten en todas las publicaciones, sin aportaciones relevantes que pudieran arrojar más luz sobre la biografía de Salgado.
} 
nombrado cura y vicario en Bragado (al oeste de la provincia de Buenos Aires) gracias a sus excelentes dotes de orador. En esta zona consiguió convertir al catolicismo a los indios que allí habitaban, así como a otros indígenas de tribus lejanas. Aparte de su labor evangelizadora, José María Salgado obtuvo varios diplomas honoríficos por parte de diferentes sociedades científicas y literarias, tanto españolas como extranjeras. Llegó a desempeñar, asimismo, el cargo de catedrático en el Liceo Universitario de Montevideo.

Con estos apuntes biográficos se descubre a Salgado como un incondicional protector de sus compatriotas, uno de los más activos y entusiastas fundadores del gran Hospital Español de Buenos Aires, además de un destacado miembro de las Sociedades de Socorros Mutuos Españolas y de la Comisión “Auxilios a España". Su casa, tanto en Buenos Aires como en Montevideo, estaba abierta a todos los emigrantes españoles, a quienes hospedaba y se preocupaba por su subsistencia (e incluso en el caso de los más necesitados, les proporcionaba el pasaje de regreso a España). Como presidente de la Comisión de los españoles, enviaba valiosas ofrendas a la patria, recibiendo, por ello, el agradecimiento de Su Majestad la reina gobernadora. En 1890 Salgado regresó a España, donde siguió ejerciendo su cometido benéfico.

En el año 1893 instauró en A Coruña una escuela dedicada a la enseñanza de los niños pobres. En dicha fundación habían cooperado dos jóvenes sacerdotes y un grupo de adolescentes que contaban con la autorización de sus padres — dada su corta edad - para encargarse de la enseñanza de los escolares. Dos años después, en 1895, se destinó en este establecimiento un departamento a la instrucción de los ciegos. La apertura del nuevo local de enseñanza fue anunciada en la prensa, al igual que el lugar y horas para hacer efectiva la matrícula.

El director de la Escuela gratuita del Campo de la Leña, D. José
María Salgado, tiene la gran satisfacción de manifestar al respeta-
ble público de La Coruña, en general, y en particular a los señores
socios protectores que desde principios de septiembre próximo y
en el mismo local de la Escuela se abrirán clases de educación e
instrucción primaria para niñas y niños ciegos, mayores de ocho
años y menores de dieciséis. Los que tengan interés en aprovechar-
se de lo que ahora sólo pobre y modestamente se ofrezca pueden
concurrir a matricularse los jueves y domingos a la calle Orzán
núm. $5,2^{\circ}{ }^{\circ}$ de once a doce de la mañana ${ }^{23}$.

23 "Obra de Caridad", El Anunciador: diario de La Coruña y de Galicia, 13705 (14 de agosto de 1895) pág. 3. 
El acto de inauguración de la escuela se celebró el día 12 del mes de octubre, a las once de la mañana, en el Campo de la Leña, número 8. Presidían la mesa destacadas personalidades de la ciudad, como el gobernador civil, D. José Pérez Ballesteros, director del Instituto, el secretario de la Escuela de Bellas Artes, y otros representantes de la prensa, del clero o del comercio. En dicho acto, tras la ejecución de una obra — al piano y a la flauta por parte de dos jóvenes ciegos-, José María Salgado, director de la Escuela, pronunció un breve discurso en el que dio a conocer el propósito de dicha fundación.

La Escuela contaba en sus inicios con siete alumnos. Además, se encargaba de que los adultos invidentes recibiesen la instrucción básica, pero priorizando la música ${ }^{24}$. Cabe precisar que la música era considerada la ocupación más apropiada para los ciegos, al considerarlos incapaces para el trabajo manual u otras artes que fuesen ajenas a la música. "La figura del ciego músico vagabundo es muy recurrente, y será precisamente esa imagen la que los nuevos maestros de los niños ciegos quieran romper" 25 .

El progreso de los alumnos de la Escuela es llamativo, con tan solo unos meses de instrucción. Si bien el Colegio de sordomudos y ciegos de Santiago de Compostela, que llevaba unos años funcionando, había alcanzado un gran reconocimiento, a la Escuela de ciegos de A Coruña tampoco le faltaban elogios:

\begin{abstract}
Bien puede decirse que Galicia va ya a gran altura en esa enseñanza que ya hizo famosa a la Escuela de Santiago y no tardará en suceder lo mismo con la de la Coruña si sigue por el camino emprendido ${ }^{26}$.
\end{abstract}

\title{
UN AMBICIOSO PROGRAMA DE ENSEÑANZA ESPECIAL
}

El director de la Escuela de ciegos aspiraba a proporcionar a sus alumnos una instrucción que estuviese a la altura del — por entonces y hasta hace muy pocos años- centro de referencia de la educación especial en España: el Colegio de sordomudos y ciegos de Madrid. A medida que transcurría el tiempo, el programa de enseñanza se fue ampliando y perfeccionando. Así, en el año 1903, los

\footnotetext{
${ }^{24}$ En la sección Notas regionales: "inauguración de la escuela de ciegos en el local de la gratuita, Campo de la Leña, núm. 8", Revista gallega: semanario de literatura e intereses regionales, 32 (20 de octubre de 1895), pág. 5.

${ }^{25}$ Esther Burgos Bordonau, "Aproximación histórica al estudio del Colegio Nacional de Sordomudos y Ciegos de España", Revista Complutense de Educación, 16, 1 (2005), págs. 183-193.

${ }^{26}$ En la sección "Locales y provinciales", El Lucense: diario católico de la tarde, 3554 (28 de septiembre de 1896) pág. 3.
} 
estudios de geografía se hicieron más extensos. Al establecimiento llegó nuevo material: una esfera de metal dedicada a la enseñanza de los ciegos que se había adquirido en un viaje a París y se había donado a la Escuela. En ese mismo curso se ensayó un nuevo sistema de escritura, utilizando unas falsillas que también habían sido compradas en el extranjero y donadas a la escuela ${ }^{27}$. Una revisión de la normativa y el plan de estudios, del año 1904, permitió comprobar la organización, la complejidad y extensión del programa de enseñanza.

Según consta en el Reglamento Interior del Establecimiento ${ }^{28}$, para ser admitido en el departamento de ciegos era condición esencial carecer del sentido de la vista y tener cumplidos los siete años (art. $3^{\circ}{ }^{\circ}$ ). En cuanto a las formalidades de ingreso, los interesados debían entrevistarse con el director (art. 5. ${ }^{\circ}$ ). Durante todo el curso, el horario de clase se extendía por las mañanas de 9:00 a 12:00, y por la tarde, de 14:00 a 17:00 (art. 6. ${ }^{\circ}$ ). Toda infracción a lo prescrito en el artículo anterior quedaba sujeta a las amonestaciones correspondientes y penitencias proporcionadas en caso de reincidencia (art. . $^{\circ}$ ). Los alumnos estaban obligados a velar por el buen estado de conservación de los libros y útiles que se le entregasen, salvo los naturales deterioros ocasionados por el uso (art. 8..$^{\circ}$ ). Cuando deliberadamente faltaran a lo prescrito en el artículo anterior, estaban obligados a reponer lo destruido (art. 9. ${ }^{\circ}$ ); lo mismo sucedería si se produjeran desperfectos en el cualquier utensilio del establecimiento o cualquier material científico (art. $100^{\circ}$ ).

Los alumnos debían presentarse a las clases diariamente aseados. Un aseo inadecuado era motivo para no dejar entrar al niño en clase, enviándolo nuevamente a casa para que se aseara debidamente (art. $\left.11 .^{\circ}\right)$. Si un alumno acumulaba ocho faltas injustificadas de asistencia a clase, en el transcurso de un mes se le enviaba a los padres o encargados un aviso de expulsión (art. 12. ${ }^{\circ}$ ). Se anularía la matrícula del alumno si después de recibido el aviso anterior, faltara ocho días más, voluntariamente, en el mismo mes (art. 13. ${ }^{\circ}$ ). Uno de los castigos aplicado a los alumnos era la reclusión en la Escuela, fuera del horario escolar. Los padres no deberían interferir en esta norma disciplinaria (art. 14. ${ }^{\circ}$ ). Los niños tenían prohibido salir de la clase dentro del horario escolar, salvo que esta salida fuera necesaria a juicio del director o profesores que estuviesen a cargo del alumno $\left(\operatorname{art} .15 .^{\circ}\right)$.

El personal del Centro debía cumplir con su cometido. Era obligación de los profesores permanecer al frente de las clases en horas de enseñanza, y si alguno

\footnotetext{
27 "Escuela de ciegos", La Correspondencia gallega: diario de Pontevedra, 4085 (1 de enero de 1903), pág. 2.

${ }^{28}$ AHUS, Fondo Universitario: colexios privados, Caixa, SH 228, Expediente 19, Escuela gratuita de ciegos y niños pobres, 1904.
} 
de los alumnos hubiera sido castigado con su reclusión en horario extraescolar, el profesor debería igualmente permanecer en el local (arts. $18 .^{\circ}$ y $19^{\circ} .^{\circ}$ ). En caso de que un profesor, por motivo justificado, no pudiese asistir, debería avisar de inmediato al director, para que encontrase con la mayor brevedad un sustituto $\left(\operatorname{art} .21 .^{\circ}\right)$.

Al portero le correspondía la tarea de asear las salas, y debía permanecer en el local a la hora de entrada y salida de los alumnos. Estaba obligado, igualmente, a cumplir las órdenes que el director o el profesorado le dictasen en beneficio de la orden y organización de la Escuela (art. 20. ${ }^{\circ}$ ). Cabe destacar que aparte del director, profesores y portero, otros profesionales prestaban servicios gratuitamente a la Escuela; este era el caso de un médico oculista.

En cuanto al sistema disciplinario que se contempla en la Escuela, los premios consistían en ascenso de puestos a las diversas secciones, vales de asistencia, aseo, aplicación, comportamiento o elogio privado y público, y carta de satisfacción a los padres o encargados de los alumnos. Los castigos consistían en la pérdida de puestos preferentes en las secciones, pérdida de vales de premio, represión privada y pública, reclusión de una o dos horas después de clase con recargo de trabajo-, y aviso de la mala conducta del alumno a sus padres o encargados. En casos más graves, el alumno podía ser expulsado temporal o definitivamente.

Los meses de vacaciones eran los de julio y agosto. Todos los días laborables, los niños debían acudir a las clases, exceptuando los festivos. Además de los establecidos por la Iglesia, se consideraban días no laborales las tardes de los jueves, el santo de su majestad el rey, fiesta cívica en A Coruña, y los días de navidad que iban del 24 de diciembre al 2 de enero (arts. $16 .^{\circ}$ y $17 .^{\circ}$ ). En las fechas navideñas se celebraban fiestas en la Escuela ${ }^{29}$. Al evento acudía alguna autoridad en representación del Ayuntamiento de la ciudad. Dicha personalidad pronunciaba un discurso ante el público asistente, recordando a los niños su deber de comportarse adecuadamente y de aprender, y les obsequiaba con mazapanes, turrones y otras golosinas. El director también dedicaba a los niños unas palabras. Estos también participaban, dando las gracias por los obsequios al Ayuntamiento y agradeciendo la educación que recibían diariamente tanto al director y a los profesores como a las entidades protectoras de la Escuela. El agradecimiento lo demostraban a través de trabajos escolares escritos en gallego, como este romance, leído por Ramón Puga, uno de los niños ciegos:

\footnotetext{
29 "Fiesta escolar", Revista gallega: semanario de literatura e intereses regionales, 406 (28 de diciembre de 1902), págs. 3-4.
} 
Día é solene ó de hoxe porque en todo o Universo celébrase o Natalicio do Fillo dun Dios Eterno. Os anxos entoan hinos aló nas rexiós do $\mathrm{Ceo}$, é os homes, acó na terra mostran ledicia e contento, e para ben demostralo cantan ao son do pandeiro facendo sonar a gaita cónchegas, fraútas e ferros. Mais hay probes que non gozan d'este Universal festexo, e se atopan neste caso moitos infelices nenos que o mesmo que os d'esta escola non lles abonda o diñeiro. Pro co a sua caridade, o noso ilustre Concello non quer que hoxe, aquí, na Cruña hacha ningún descontento de sorte que, pra lograr o seu manánimo ouxeto, mandoúnos á nosa casa dóces, tourróns, caramelos e outras moitas larpeiradas que co a yalma agradecemos. ¡Dios pague tanta bondade de quen lembra aos pobres nenos, pra facerlles comprender que na vida non son menos que os ricos, pos son hirmáns según reza ó Evanxelio! E o Nenillo que en Belém entre humildades nasceo poidendo ter pra servilo aos anxeliños do ceo, derrame todalas gracias sobre de quen fai o ousequio 
que recibimos sintindo

cheo de amor noso peito.

E faga tamén que a Escola

de coitados pobres cegos

teña vida e aumente moito,

pra rendir o xusto premio

ao seu dino Direutor

que é pai d'estes pobres nenos.

El plan de estudios era muy completo. Formaban parte del programa de enseñanza la lectura, escritura, doctrina cristiana, historia sagrada, aritmética, gramática castellana, historia de España, higiene, geometría, geografía práctica, solfeo, armonía, piano, órgano, violín, guitarra y flauta ${ }^{30}$ :

La escuela contaba con material específico ${ }^{31}$ para uso de los alumnos. Disponía de diferentes cajas, de cubos aritméticos (sistema Llorens), cajetines de resolución de problemas, cajas de aritmética (sistema español), esfera terrestre, mapas de España y Europa en relieve, falsillas para escribir con lápiz, distintos instrumentos musicales (flauta, violín, piano, contrabajo y guitarra), pautas del sistema Llorens, Braille, Avreu y Balú. La Escuela también reunía obras literarias, en el sistema convencional, de autores célebres, como Julio Verne, Duque de Rivas, Cervantes, Pereda o Zorrilla, y de contenido musical, como tratados de solfeo y piano.

Los exámenes finales se realizaban antes de las vacaciones veraniegas. Se recoge a continuación un programa de ejercicios ${ }^{32}$ propuesto para el examen de los alumnos ciegos, de los días 16, 17 y 18 de junio de 1904. Se examinaban en horario de mañana y tarde, de 10:30 a 13:00 y de 17:00 a 19:00.

1. Preludio de la velada núm. 1 (Caño) para violines, piano y contrabajo, efectuado por los alumnos Ramiro Puga, Ángel Gómez y José Villar, acompañados por el profesor.

2. Principios de lectura en el sistema convencional de Braille y en el usual relieve, por las niñas Luisa Gutiérrez y Claudina Fernández, y los alumnos Ramón Rey, Juan Prego y Ramiro Puga.

3. El eclipse de sol. Melodía para violín y piano (Raf), por los alumnos Ramiro Puga y José Villar.

\footnotetext{
${ }^{30}$ AHUS, Fondo Universitario: colexios privados, Caixa, SH 228, Expediente 19, Escuela gratuita de ciegos y niños pobres, 1904 .

${ }^{31}$ Ídem.

${ }^{32}$ Ídem.
} 
4. Escritura convencional en el sistema Braille, por los alumnos Juan Prego y Ramiro Puga. Ídem, usual con lápiz por Ángel Gómez y José Longueira.

5. Marcha de la coronación en la opera profeta (Meyerbeer), ejecutada a cuatro manos por el alumno José Vilar y su profesor Sr. Caño.

6. Ejercicios teórico-prácticos de la Aritmética con resolución de problemas en la caja y el tablero especial por los alumnos Ramón Rey, Juan Prego y José Villar.

7. Escena y aria final de la ópera Luccia de Lamermoor (Donizeti), ejecutada al violín por el alumno Ángel Gómez.

8. Conocimientos generales sobre la esfera terrestre, por los alumnos Ramón Rey, Juan Prego y José Villar. Ídem, particulares sobre el mapa de Europa por el alumno Ramiro Puga. Ídem geográfico históricos sobre el mapa de España por los alumnos Ramón Rey, Juan Prego y José Villar.

9. Ejecución al piano de una sonatina de Clementi, por el alumno José Villar.

10. Conocimientos de Gramática, Religión, Historia y Ciencias físico-naturales con descripción de objetos, por las niñas Luisa Gutiérrez y Claudina Fernández, y los alumnos Ramón Rey, Juan Prego, José Villar y Ramiro Puga.

11. Adelante, galop-capricho para piano a cuatro manos (Caño), ejecutado por el alumno José Villar y su profesor.

12. Lecturas de poesías y trozos escogidos de autores clásicos, por los alumnos Ángel Gómez y Ramiro Puga.

13. Himno cantado por todos los alumnos de ambos sexos, acompañados al piano por la Señorita Lola Infante Rodríguez Recio.

14. Terminará el acto con la lectura de una memoria hecha por el Sr. Secretario.

\section{UN ITINERARIO EXITOSO ACOMPAÑADO DE PENURIAS Y ESCASEZ}

La Escuela de ciegos de Salgado se mostró eficaz en su cometido desde el primer mes de su inauguración. La publicación madrileña La Lectura dominical testifica lo dicho:

Se ha creado en la Coruña una escuela gratuita de ciegos. Uno de tantos obscurantistas de sotana y manteo, el caritativo Sacerdote D. José María Salgado, es el fundador. Como institución de verdadera caridad, no se limita el número ni la edad de los admisibles. Ser ciego es suficiente condición para el ingreso. Un mes lleva la expresada escuela, y ya la prensa de la Coruña se hace lenguas de los resultados obtenidos. Bien digna es la obra del presbitero 
señor Salgado de la protección de las corporaciones populares de la Coruña y aun de la provincia ${ }^{33}$.

Los exámenes se celebraban públicamente en el local del establecimiento, lo que permitía demostrar a los asistentes las destrezas y habilidades adquiridas por los alumnos en las diferentes asignaturas. Por otra parte, el progreso de los escolares impulsaba a los socios protectores a continuar sufragando los gastos de la Escuela, así como al Ayuntamiento y corporaciones provinciales.

El Tribunal que presidía las pruebas estaba formado por distintas autoridades (entre otros, el gobernador civil, representantes del ayuntamiento y el inspector de enseñanza de la provincia). En ocasiones, acudían a la convocatoria otras personalidades destacadas del extranjero, como algún capitalista procedente de Buenos Aires.

Los alumnos ciegos realizaban ante el público las pruebas de escritura, lectura, contabilidad, solfeo, instrumento y otros conocimientos, efectuándolas con facilidad y brillantez, cosa que evidenciaba, además de la inteligencia de los niños, la entrega, paciencia y competencia del director y de los profesores. Entre el profesorado, se destacaba el maestro ciego Luciano Caño, que además de sus buenas dotes como profesor era un excelente compositor de música y dominaba varios instrumentos.

Los exámenes finalizaban con la felicitación de las autoridades presentes. Se elogiaba la labor de enseñanza llevada a cabo en la Escuela. El dominio de las diferencias asignaturas por parte de los niños ciegos sorprendía a los asistentes. Pero el mérito de este aprendizaje podría considerarse todavía más reseñable, debido a que muchos de los niños ciegos que asistían a las clases tenían que ocuparse en otras tareas o trabajos, lo que les restaba tiempo para el estudio ${ }^{34}$.

El progreso de los escolares no solo se evidenciaba en los exámenes realizados públicamente: Salgado aprovechaba cualquier festival u homenaje para demostrar la formación que habían adquirido sus discípulos. Asimismo, el director acudía con sus alumnos a diferentes exposiciones y concursos.

Es importante destacar la función que desempeñaron estas exposiciones en el desarrollo de las técnicas, métodos y procedimientos para la mejora en la instrucción de personas ciegas y sordomudas. Las exposiciones eran un buen

\footnotetext{
$\overline{{ }^{3} 3}$ En la sección "noticias y comentarios", La Lectura dominical, 102 (15 de diciembre de 1895), pág. 12.

34 "Escuela de ciegos de la Coruña", Revista Gallega: semanario de literatura e intereses regionales, 120 (27 de junio de 1897), pág. 4.
} 
medio de divulgación, que permitían probar los nuevos adelantos y avances en la materia ${ }^{35}$.

El éxito obtenido en estos certámenes se convertía en alabanzas a la enseñanza practicada en el Escuela y al director, al que se le otorgan calificativos como estos: "Ese hombre de corazón magnánimo, ese caritativo sacerdote honra de su clase, ese incansable maestro y protector de los que no ven, se llama Don José María Salgado ${ }^{36}$ ". En este sentido, se descubre en la prensa alguna anécdota que deja entrever la personalidad caritativa de Salgado, así como el trato cariñoso que dispensaba a los escolares. Una nota del periódico El áncora ${ }^{37}$ testimonia que José María Salgado regaló unos abrigos a dos de sus alumnos ciegos acogidos en el asilo, rogando al alcalde, además, que les permitiese estrenarlos el día 23 (un día señalado, probablemente para lucirlos en algún acto navideño de la escuela). Se recogen a continuación los elogios recibidos en la Exposición Regional celebrada en la ciudad de Lugo:

Aún nos parece que era ayer cuando en presencia de una concurrencia doctísima, nos daba a conocer los prodigios que obra la caridad, cuando esta lleva por cortejo la ilustración y la paciencia. El ver como aquellos ciegos, con seis meses escasos de preparación, contestaban sin dudas ni vacilaciones, a las preguntas de Gramática, Geografía, Aritmética, Geometría, Escritura, etc., etc., producía en nuestro ánimo, un entusiasmo tal, que momentos hubo en que no podíamos contener las lágrimas ni el movimiento de nuestros brazos para estrechar al que tanto bien hace. Imponiéndose el sacrificio de su caudal, de su bienestar y de una paciencia sometida a durísima prueba. Y no sólo en los ejercicios de dichas asignaturas hemos admirado los resultados de la obra realizada por el Sr. Salgado, sino que también en la enseñanza musical hemos quedado altamente complacidos. Bien puede decirse, sin que en ello pequemos de exagerados, que en el Sr. Salgado obra el prodigio de que vean los que del órgano de la vista, carece. Y si esto hace, con sólo seis meses de instrucción ¿Qué no será cuando

\footnotetext{
${ }^{35}$ Esther Burgos Bordonau, Historia de la enseñanza musical para ciegos en España, 18301938, Madrid, ONCE, 2004, pág. 60.

${ }^{36}$ El Eco de Galicia: diario de la tarde, núm. 3156 del 12 de diciembre de 1896, en su pág. 1, se dedica un largo escrito a la escuela de ciegos de Salgado, elogiando su participación en la Exposición Regional celebrada en la ciudad lucense. En este artículo, titulado "Recuerdos de la exposición. Escuela de ciegos de la Coruña", se recoge también la biografía del Sr. Salgado.

37 "De la Región. Coruña", El áncora: diario católico de Pontevedra, 205 (23 de diciembre de 1897), pág. 2.
} 
los ciegos discípulos, puedan ampliar sus conocimientos en dos o tres años? ${ }^{38}$

El director de la Escuela también acompañaba a los niños a festivales convocados fuera de Galicia, como la exposición regional de Gijón. En esta ocasión, Salgado viajó a la ciudad asturiana con ocho de los alumnos ciegos. Dos de ellos pertenecían al asilo local, y uno, al hospicio provincial ${ }^{39}$.

La Escuela recibió premios en varios certámenes. En el concurso musical celebrado en Santiago de Compostela en el año 1906, los dos primeros premios les fueron otorgados a los alumnos. El director se veía recompensado con el máximo galardón, también, en dichos eventos. Pero lo cierto es que pese al reconocimiento de su eficiente labor, el funcionamiento del establecimiento estuvo marcado por la penuria y la escasez. En cualquier caso, esto era lo habitual en los centros y colegios de finales del siglo XIX y principios del $\mathrm{XX}^{40}$.

La Escuela, con el paso del tiempo, se hacía más difícil de sostener. El déficit se acumulaba año tras año. Era preciso adquirir material especial para la educación de los ciegos. Para su instrucción no bastaba con un silabario o un lápiz, como sucedía con los alumnos videntes, sino que precisaban de aparatos especiales y costosos que, por entonces, eran escasos. El material en la enseñanza especial, tal como apunta el director del Colegio Regional de Santiago de Compostela, López Navalón, era parte fundamental de la enseñanza, constituyendo un importante apoyo para que los alumnos consiguiesen formarse una idea del mundo externo. Este material específico les proporcionaba una base para cimentar sobre ella todos los conocimientos que gradualmente deberían ir adquiriendo durante el período que durase la enseñanza.

Si fuera posible que esos Establecimientos poseyesen el mundo en pequeño, que la naturaleza estuviera representada por pequeños modelitos, que los progresos humanos en todas sus esferas se presentasen materialmente, que todo cuanto es del dominio del hombre lo vieran tangiblemente, la instrucción de estos desgraciados

\footnotetext{
38 "Recuerdos de la exposición. Escuela de ciegos de la Coruña", El Eco de Galicia: diario de la tarde, 3156 (12 de diciembre de 1896), pág. 1.

${ }^{39}$ En la sección "Noticias de Galicia", Gaceta de Galicia: diario de Santiago. Decano de la prensa de Compostela, 158 (7 de julio de 1899), pág. 2.

${ }^{40}$ Isabel GutiérRez Zuloaga, Introducción a la historia de la logopedia, Madrid, Narcea, 1997, pág. 119.
} 
sería tan completa y tan perfecta como la de los que poseen todos sus sentidos ${ }^{41}$.

La creatividad y la habilidad de Don Manuel López Navalón permitirían a los alumnos ciegos del Colegio de Santiago disfrutar del material específico para sus clases. Sin embargo, José María de Salgado no contaba con las mismas dotes creativas y en la ciudad de A Coruña: no existían aparatos y otros útiles necesarios que la escuela precisaba. Había que solicitarlos a la capital, Madrid, o incluso al extranjero, lo que encarecía mucho la compra.

A la necesidad de material para los escolares ciegos se sumaba el alquiler de local, el sueldo de los profesores y auxiliares y otros gastos de menor cuantía. Es preciso señalar que, por entonces, solo el Colegio de sordomudos y ciegos de Madrid se financiaba con los fondos del Estado ${ }^{42}$. La situación llegó a ser tan desesperante, que D. José M. Salgado acordó la publicación y propagación de unas cartas para que los interesados pudiesen colaborar a que la Escuela se mantuviese activa:

Siendo cada día de mayor consideración los gastos que se originan para atender cumplidamente a cuanto exige la índole de este Colegio, y muy escasos y eventuales los recursos de que por razón de ingresos puedo disponer resultando por tanto un crecido déficit cada año, me veo en la imprescindible necesidad de recurrir a los bondadosos y nobles sentimientos que a usted distingue, rogándole se sirva subscribir la adjunta nota con lo que su voluntad le dicte, para el mejor sostenimiento, perfección y progreso de este centro educativo ${ }^{43}$.

Era una escuela necesaria, al ser la única institución de este tipo en la ciudad. Tal como aseguraba el diario madrileño La Unión Católica ${ }^{44}$, esta escuela prestaba un servicio importante. Eran pocos los ciegos que en los últimos años del siglo XIX y principios del XX podían acceder a la instrucción y a la protección

\footnotetext{
${ }^{41}$ Manuel López Navalón, Memoria sobre el estado del Colegio de Sordo-Mudos y de Ciegos de Santiago y reformas que deben plantearse para su mejor organización, Santiago de Compostela, Imprenta Manuel Miras, 1870, pág. 43.

${ }^{42}$ E. Burgos Bordonau, Historia de la enseñanza musical..., pág. 170.

43 "La escuela gratuita de ciegos y niños pobres", Revista gallega: semanario de literatura e intereses regionales, 353 (22 de diciembre de 1901), pág. 4.

${ }^{44}$ En la sección "Culto y Clero", La Unión Católica: diario religioso, político y literario, 2535 (21 de diciembre de 1895), pág. 2.
} 
oficial necesaria para garantizar su subsistencia. Para remediar este abandono social, comenzaron a emerger diferentes organizaciones y patronatos.

En el año 1894, se inauguró el Centro instructivo y protector de ciegos en Madrid. Esta institución se ocupaba no solo de la instrucción de los ciegos, sino también de protegerlos en caso de necesidad. "Era una sociedad de socorro mutuo y de resistencia contra las campañas de mendicidad"45. En enero de 1907, se celebró en Madrid la Asamblea Nacional para el Mejoramiento de la Suerte de los Ciegos y los Sordomudos, con la presencia de varios representantes del Centro instructivo y protector de ciegos. A raíz de las resoluciones de dicha Asamblea, se creó por Real Decreto de 22 de enero de 1910 el primer Patronato nacional de ciegos, sordomudos y anormales. Los ciegos y sordomudos gallegos presentaron una instancia al ministro de instrucción pública, en la que manifestaban su acuerdo con dicho Decreto y solicitaban que se llevara a efecto ${ }^{46}$.

En septiembre de 1910, solventadas ciertas dificultades que retrasaban su establecimiento, se constituyó definitivamente el Centro instructivo y protector de sordomudos y ciegos de Galicia. El profesor de la escuela de Salgado, D. Luciano Caño, formaba parte de la junta directiva, ostentando el cargo de vicepresidente primero ${ }^{47}$. En este Centro podían matricularse los ciegos de ambos sexos de toda Galicia. A los residentes en A Coruña les bastaba con enviar una solicitud a la junta directiva de este para hacer efectiva la matrícula. Los de fuera de la localidad, además de la solicitud, tenían que remitir un certificado de buena conducta visado por el alcalde o cura párroco del punto de residencia ${ }^{48}$. En diciembre de ese mismo año se dispusieron los talleres en la calle Panaderas para los ciegos que se instruyesen en dicha asociación ${ }^{49}$.

Pero esta asociación, según denuncias que aparecen en la prensa, no funcionaba debidamente, observándose ciertas irregularidades en su actividad ${ }^{50}$. Esta

\footnotetext{
${ }^{45}$ E. Burgos Bordonau, Historia de la enseñanza musical..., pág. 164.

${ }^{46}$ Ciegos y sordomudos gallegos. Una instancia”, El Correo de Galicia: diario independiente de avisos y noticias, $(27$ de abril de 1910) pág. 2.

${ }^{47}$ En la sección Noticias de Galicia-Coruña. La Correspondencia gallega: diario de Pontevedra, 6169 (19 de setiembre de 1910), pág. 1.

${ }^{48}$ En la sección el día en la Coruña en El Eco de Galicia: diario católico é independiente, 1227 (27 de agosto de 1910), pág. 3.

${ }^{49}$ En la sección noticias del Diario de Galicia: periódico de la mañana, telegráfico, noticiero y de información general: 615, 17 de diciembre de 1910 y "El día en la Coruña", El Eco de Galicia: diario católico é independiente, 1323 (16 de diciembre de 1910), pág. 3.

${ }^{50}$ El presidente del Centro instructivo y protector de sordomudos y ciegos, el Sr. Izquierdo, apoyado por otros miembros de dicha asociación, responde a las acusaciones que aparecen en la prensa negando que dicha Sociedad, haya cometido ningún tipo de irregularidad en su actividad. El Eco de Galicia: diario católico é independiente 124 (13 de septiembre de 1910), pág. 1.
} 
situación se expone con mucha claridad en la carta que aparece en el periódico el Eco de Galicia, firmada por algunos de los miembros de dicha Sociedad:

Muy Señor Nuestro: Rogamos a usted la inserción, de las siguientes líneas en su ilustrado diario, suplicándole nos perdone la molestia que con tal motivo nos vemos obligados a ocasionarle.

En el corto tiempo que hemos pertenecido al "Centro Instructivo y protector de ciegos" actualmente en la calle de Panaderas hemos adquirido la plena convicción de que más que una Sociedad filantrópica, se perseguían en él, miras egoístas por algunos de los individuos. Así lo hemos hecho constar en los diarios locales en el mes de setiembre último. Posteriormente los hechos comprobaron la exactitud de nuestras afirmaciones, como lo demuestra la protesta que hace poco apareció en la prensa firmada por algunos ciegos, en la que se hacían constar cargos contra el presidente de los mismos, Sr. Izquierdo ${ }^{51}$.

Asimismo, en dicha notificación se acusaba a esta Sociedad de crear escuelas para educar a los ciegos y sordomudos del hospicio sin reunir las condiciones requeridas. No estaban dotadas del material específico necesario para la enseñanza, ni contaban con profesorado capacitado.

Mas lo verdaderamente inconcebible es que de esta sociedad de cuyo seno se han apartado todos los faltos de vista protestando contra la explotación de que eran víctimas, que esta sociedad repetimos se atrevió a solicitar de la Excma. Diputación, en términos poco respetuosos, por cierto, que los ciegos y sordomudos acogidos en el Hospicio provincial, ingresen en las escuelas que dicen haber creado. Hasta hoy solo tienen estas escuelas un local de bastante medianas condiciones pedagógicas a la puerta del cual se ve una flamante rótulo, no poseen absolutamente ningún material adecuado para los seres que pretenden instruir, carecen de profesorado para sordomudos y el que pretende ponerse al frente de la enseñanza de ciegos es un joven inexperto expulsado por sedicioso de la Casa de Misericordia, del Asilo Municipal y de la Escuela gratuita de ciegos y niños pobres que en el Campo de la

\footnotetext{
51 "Una Carta", El Eco de Galicia: diario católico é independiente, 1361 (31 de enero de 1911), pág. 1 .
} 
Leña tiene establecida el Sr. Salgado desde hace dieciocho años. Tal es la verdad de los hechos ${ }^{52}$.

Luciano Caño, vicepresidente de dicha Sociedad, firmó esta denuncia junto con otros miembros del Centro instructivo de ciegos y sordomudos. Caño se convirtió, un año después de estas denuncias, en el presidente del Centro instructivo de ciegos de A Coruña, una sociedad análoga a la establecida en Madrid, constituida con motivo de la visita a la ciudad gallega de Eugenio Canora, viejo líder de las asociaciones de ciegos y vocal de Patronato de sordomudos, ciegos y anormales. Canora, como comisionado de dicho Patronato, había viajado por algunas ciudades españolas del norte y levante ${ }^{53}$ para comprobar el estado en que se encontraban las instituciones benéficas que impartían la enseñanza a los ciegos y los medios de que disponían estos para sobrevivir. "Aprovecharía, además, este viaje, para solicitar una credencial al Centro Instructivo de Madrid, que le hiciera representante oficial del mismo ante otras asociaciones de ciegos" ${ }^{54}$. Con su visita a A Coruña, en agosto de 1912, quedaría establecido el Centro de ciegos de la ciudad:

El presidente del Centro instructivo y protector de ciegos de Madrid, D. Eugenio Canora, ha construido en la Coruña una entidad análoga que será sucursal de la central, declarada hace poco de Real Orden Sociedad benéfica.

De acuerdo a las ordenanzas de dicha Sociedad, los niños se instruirían en la escuela de ciegos de Salgado. "Los niños recibirán enseñanza en la escuela de ciegos de Salgado, que así lo ha ofrecido galantemente" ". Cabe señalar que en su estancia en la ciudad gallega, Canora había pronunciado una conferencia que versaba sobre la enseñanza de los ciegos en esta Escuela. A las niñas se les

\footnotetext{
52 "Una Carta", El Eco de Galicia: diario católico é independiente, 1361 (31 de enero de 1911), pág.1.

${ }^{53}$ Los detalles de su viaje se pueden leer en la memoria que dejó escrita. Eugenio Canora MoleRo, Memoria explicativa del viaje de estudio hecho a las provincias de Alicante, Valencia, Barcelona: Zaragoza, Bilbao, Santander y Coruña, Madrid, Imprenta del Colegio Nacional de Sordomudos y de Ciegos, 1913.

${ }^{54}$ Roberto Garvía Soto, La Organización Nacional de Ciegos: un estudio institucional, Madrid, Centro de Estudios Avanzados en Ciencias Sociales, 1992, pág. 38.

55 "Un centro de ciegos", El Diario de Pontevedra: periódico liberal, 8491 (30 de agosto de 1912), pág. 2. Sobre la visita de Canora a la ciudad A Coruña también se puede revisar, en la sección de las "Noticias regionales, Coruña", la Gaceta de Galicia: diario de Santiago. Decano de la prensa de Compostela, 195 (29 de agosto de 1912) pág. 2.
} 
enseñaría en otro establecimiento, cedido por la Junta Provincial de Protección a la Infancia.

Pero los donativos que llegaban al Centro de ciegos de A Coruña del Patronato de sordomudos, ciegos y anormales ${ }^{56}$, la contribución del ayuntamiento y de la diputación provincial y las ayudas de algunos filántropos protectores continuaban siendo insuficientes para cubrir los gastos de la Escuela. La revista gallega Gaceta de Galicia, así como otras publicaciones de la provincia, se hacían eco de la falta de recursos y de la necesidad de fondos para su mantenimiento. La prensa también estaba atenta a los donativos, e incluso notificaba el nuevo material, ya fuese el cedido a la Escuela o el adquirido por el director. En el año 1915, la escuela recibirá como donativo un planímetro, construido en el colegio de sordomudos y ciegos de Santiago de Compostela, y cuatro tomos de geometría $^{57}$. El director se mostraba agradecido ante cualquier obsequio útil que contribuyese a mejorar la enseñanza; ahora bien, Salgado también manifestaba su resentimiento cuando consideraba que a la Escuela se la excluía del reparto de donativos efectuado por organismos o asociaciones con fines benéficos:

Me interesa hacer constar que en la repartición de donativos efectuada por la sociedad Reunión Recreativa e Instructiva de artesanos a favor de la entidades protectores de los niños pobres, la escuela gratuita de ciegos y niños pobres, a mi cargo, sita en el Campo de la Leña número 8 (quizá por olvido involuntario) no ha recibido absolutamente ni el más pequeño donativo. $Y$ digo por olvido porque a no obedecer a dicha causa no se explica que sea preterida una escuela tan conocida por sus fines benéficos en pro de la niñez desvalida que viene funcionando sin interrupción desde hace veintitantos años ${ }^{58}$.

En la década de los veinte, la situación seguía siendo penosa. La Junta de gobierno que ejercía el Patronato de la Escuela pretendía obsequiar a los niños en las fechas navideñas ofreciéndoles algunos regalos para premiar su aplicación al estudio, pero la escasez de recursos lo impedía. La Junta carecía de prendas de vestir, golosinas u otros obsequios para repartir a los alumnos del centro; por ello, se veían obligados a solicitar limosnas en metálico o en prendas de abrigo,

\footnotetext{
56 "Para el centro instructivo de ciegos: Un donativo", El Eco de Galicia: diario católico é independiente, 1983 (31 de enero de 1913), pág.1.

57 "La escuela de ciegos. Un donativo", El Eco de Galicia: diario católico é independiente, 2975 (23 septiembre de 1915), pág. 1.

58 "Una Carta", El Eco de Galicia: diario católico é independiente, 2943 (22 de agosto de 1915), pág. 1.
} 
para que los escolares pudiesen celebrar las festividad de Reyes ${ }^{59}$. Sin embargo, esta lamentable situación no supuso un obstáculo para que la escuela mantuviese su actividad. En la década de los treinta, a juzgar por alguna que otra reseña aparecida en la prensa, la Escuela gratuita de ciegos y niños pobres de Salgado todavía se mantendría activa ${ }^{60}$.

\section{CONCLUSIÓN}

La primera ley que hace referencia a la enseñanza de sordomudos y ciegos en España data del año 1857. A raíz de esta ley, comenzarán a establecerse colegios y escuelas de enseñanza especial para estos alumnos. Galicia contará con el primer Colegio regional que se instaura en España conforme a los dictados de dicha ley. Este establecimiento se inaugura en el año 1864 en la ciudad gallega de Santiago de Compostela. Habría que esperar más de veinte años para que se crease en Galicia otro establecimiento dedicado a la enseñanza especial, esta vez, gracias al impulso del sacerdote José María Salgado.

La inauguración de esta escuela, en la ciudad de A Coruña, supone una contribución significativa en los inicios de la educación de los ciegos en Galicia. Un digno establecimiento a incluir en los escasos centros de enseñanza especial que aparecen a finales del siglo XIX en España. Si bien se trataba de una escuela no oficial, era un establecimiento de enseñanza perfectamente constituido, con un complejo y extenso programa de instrucción y con el ambicioso propósito de conseguir para sus alumnos la misma educación que se ofertaba en el Colegio nacional de sordomudos y ciegos de Madrid (modelo, en cuestiones de enseñanza especial, hasta hace poco tiempo).

Fue un reto que se vería obstaculizado por su precaria situación económica. Aún así, la Escuela de ciegos permanecería activa durante décadas. En el año 1925, según se constata en la estadística de colegios y escuelas públicas y privadas encargadas de la educación de ciegos y sordomudos en Galicia, la Escuela de ciegos y niños pobres del Campo de la Leña, así como el Colegio Regional de sordomudos y ciegos de Santiago de Compostela, continuarían siendo las únicas instituciones que en Galicia se encargaban de la educación de estos alumnos, un dato significativo para esta escuela, que por su trayectoria merece ser rescatada del olvido a que ha quedado relegada.

\footnotetext{
59 "Escuela gratuita de ciegos y de niños pobres", El ideal gallego, 1932 (29 de noviembre de 1923), pág. 4.

60 "La escuela gratuita", El pueblo gallego, diario de la mañana, (31 de diciembre de 1933), pág. 6.
} 


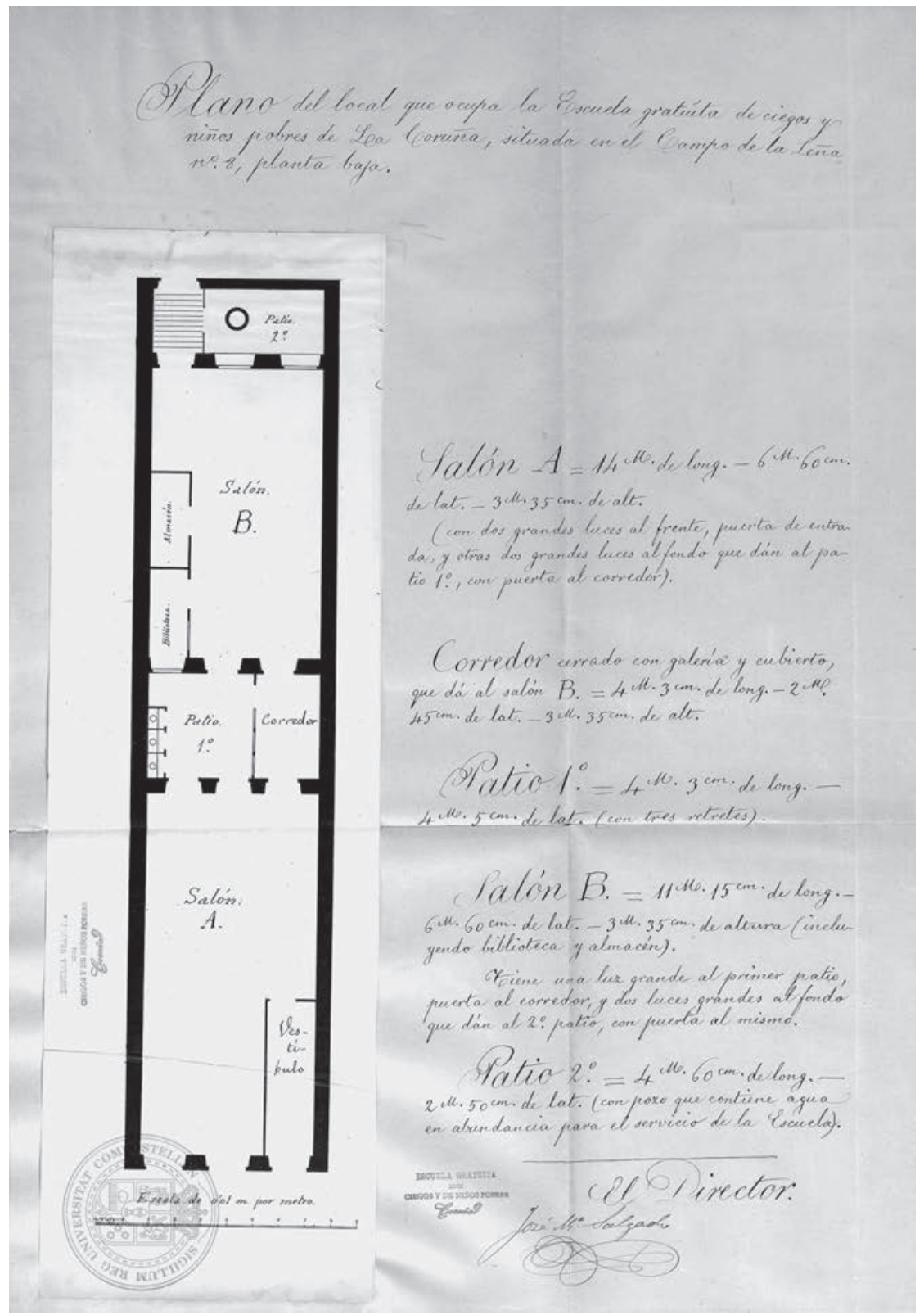

Fig. $2^{61}$

\footnotetext{
${ }^{61}$ Plano de la Escuela gratuita de ciegos y niños pobres: AHUS, Fondo Universitario: colexios privados, Caixa, SH 228, Expediente 19, Escuela gratuita de ciegos y niños pobres, 1904.
} 


\section{BIBLIOGRAFÍA}

Aguado Díaz, Antonio, Historia de las deficiencias, Madrid, Once, 1995.

Burgos Bordonau, Esther, Historia de la enseñanza musical para ciegos en España, 18301938, Madrid, ONCE, 2004.

Burgos Bordonau, Esther, "Aproximación histórica al estudio del Colegio Nacional de Sordomudos y Ciegos de España", Revista Complutense de Educación, vol. 16, 1, (2005), pág. 183-193.

Canora, Eugenio, Memoria explicativa del viaje de estudio hecho a las provincias de Alicante, Valencia, Barcelona: Zaragoza, Bilbao, Santander y Coruña, Madrid, Imprenta del Colegio Nacional de Sordomudos y de Ciegos, 1913.

Colegio de sordomudos y ciegos de Santiago de Compostela, Bases y Reglamento para el establecimiento y régimen del colegio de Sordomudos y de ciegos del distrito universitario de Santiago, Santiago de Compostela, Imprenta Manuel Mirás, 1863.

Comellas García, José L., Historia España Contemporánea, Madrid, RIALP, 2002.

Costa Rico, Antón, Historia da educación e da cultura en Galicia. (Séculos IV-XX), Vigo, Xerais, 2004.

Díaz Huertas, José A., Ruíz Díaz, Miguel A., y Ruíz Díaz, Víctor, Infancia y discapacidad, Madrid, Sociedad de Pediatría Social, 2007.

Fernández Caamaño, José M., La Coruña Vista Desde sus Libros de Actas II, Tomo II, Madrid, Editorial Visión Net, 2006, pág. 111.

Fernández Villabrille, Francisco, Estado actual y organización de la enseñanza de Sordomudos y ciegos. Memoria dirigida al Ministro de Fomento, Madrid, Imprenta del Colegio de Sordomudos, 1862.

Garvía Soto, Roberto, La Organización Nacional de Ciegos: un estudio institucional, Madrid, Centro de Estudios Avanzados en Ciencias Sociales, 1992, pág. 38.

Gascón Ricao, Antonio, "Crónica simple sobre diez años de investigación sobre las personas sordas", en Personas sordas y Universidad: el valor de la docencia y de la investigación universitarias. Conferencia presentada en las II Jornadas Universitarias organizadas por la Universidad de Sevilla, 15 de abril de 2009, Sevilla, Universidad de Sevilla, 2009, págs. 254-261.

Gabriel Fernández, Narciso de, "Financiación de la escuela pública en la España del siglo XIX. El caso Gallego", Historia de la educación, revista interuniversitaria, 7 (1988), págs. 163-178.

Gutiérrez Zuloaga, Isabel, Introducción a la historia de la logopedia, Madrid, Narcea, 1997.

Herraiz Gascueña, Mariano, Aproximación a la educación especial española del primer tercio del siglo XX, Murcia, Compobell, 1995.

López Navalón, Manuel, Memoria sobre el estado actual del Colegio de Sordo-Mudos y de Ciegos de Santiago. Mejoras de que es susceptible para su futuro progreso y prosperidad, Santiago de Compostela, Imprenta Manuel Miras, 1867.

López Navalón, Manuel, Memoria sobre el estado del Colegio de Sordo-Mudos y de Ciegos de Santiago y reformas que deben plantearse para su mejor organización, Santiago de Compostela, Imprenta Manuel Miras, 1870. 
Molina Martín, Pedro, "Los Institutos de sordomudos y ciegos", La Escuela Moderna, Revista pedagógica hispano-americana, 111 (junio de 1900), pág. 455.

Negrín Fajardo, Olegario, Historia de la educación española, Madrid, Universidad Nacional de Educación a Distancia, 2011.

Otero Urtaza, Eugenio, "O discurso de Victoriano Encinas no Congreso Pedagóxico de Pontevedra (1887)”, Sarmiento. Anuario Galego de Historia da Educación, 1 (1997), pag.119214.

Porto Ucha, Ángel Serafín, "La educación especial en la formación de maestros y maestras en el primer tercio del siglo XX. Algunos datos referentes a Galicia", en El largo camino hacia una educación inclusiva, la educación especial y social del siglo XIX a nuestros días. XV Coloquio de Historia de la Educación, Pamplona-Iruñea, Universidad de Navarra, 2009, págs. 335-344.

Puelles Benítez, Manuel, "Los valores en las grandes leyes de educación”, en Muñoz Repiso, M., Valle López, J. M., Villalaín Benito, J. L. (eds.), Educación y valores en España, Madrid, Ministerio de Educación y Ciencia, 1992, págs. 63-83.

Suárez Pazos, Mercedes, "El campesinado gallego y su rechazo a la escuela primaria (18681874)", Historia de la educación. Revista interuniversitaria, 2 (1983) págs. 317-324.

Veiga, Manuel, O pacto galego na construcción de España, Vigo, A nosa terra, 2003. 